\title{
A Modified Generalized Laguerre Spectral Method for Fractional Differential Equations on the Half Line
}

\author{
D. Baleanu, ${ }^{1,2,3}$ A. H. Bhrawy, ${ }^{4,5}$ and T. M. Taha ${ }^{5}$ \\ ${ }^{1}$ Department of Mathematics and Computer Sciences, Faculty of Arts and Sciences, Cankaya University, \\ Eskisehir Yolu 29.Km, 06810 Ankara, Turkey \\ ${ }^{2}$ Department of Chemical and Materials Engineering, Faculty of Engineering, King Abdulaziz University, \\ Jeddah 21589, Saudi Arabia \\ ${ }^{3}$ Institute of Space Sciences, Magurele-Bucharest, Romania \\ ${ }^{4}$ Department of Mathematics, Faculty of Science, King Abdulaziz University, Jeddah 21589, Saudi Arabia \\ ${ }^{5}$ Department of Mathematics, Faculty of Science, Beni-Suef University, Beni-Suef 62511, Egypt
}

Correspondence should be addressed to T. M. Taha; taha_m_taha@yahoo.com

Received 21 May 2013; Accepted 7 July 2013

Academic Editor: Soheil Salahshour

Copyright (C) 2013 D. Baleanu et al. This is an open access article distributed under the Creative Commons Attribution License, which permits unrestricted use, distribution, and reproduction in any medium, provided the original work is properly cited.

\begin{abstract}
This paper deals with modified generalized Laguerre spectral tau and collocation methods for solving linear and nonlinear multiterm fractional differential equations (FDEs) on the half line. A new formula expressing the Caputo fractional derivatives of modified generalized Laguerre polynomials of any degree and for any fractional order in terms of the modified generalized Laguerre polynomials themselves is derived. An efficient direct solver technique is proposed for solving the linear multiterm FDEs with constant coefficients on the half line using a modified generalized Laguerre tau method. The spatial approximation with its Caputo fractional derivatives is based on modified generalized Laguerre polynomials $L_{i}^{(\alpha, \beta)}(x)$ with $x \in \Lambda=(0, \infty), \alpha>-1$, and $\beta>0$, and $i$ is the polynomial degree. We implement and develop the modified generalized Laguerre collocation method based on the modified generalized Laguerre-Gauss points which is used as collocation nodes for solving nonlinear multiterm FDEs on the half line.
\end{abstract}

\section{Introduction}

Due to the fact that fractional calculus has extensive applications in chemistry, engineering, neuron modeling, and biological sciences, the use of fractional calculus has attracted great interest from the mathematical science research community (e.g., see [1-5]).

As is well known, spectral methods use orthogonal polynomials as the basis functions and so usually provide accurate numerical results, see [6-9] and the references therein. In particular, we could employ tau and pseudospectral modified generalized Laguerre approximations and interpolation to solve multiterm fractional differential equations on semiinfinite interval. However, some authors developed the modified generalized Laguerre spectral method for the half line for ordinary, partial, and delay differential equations, see $[8,10-$ 12]. The Laguerre polynomials of fractional orders and its generalization can be expressed by the Bell polynomials, see [13-15].

Because of the difficulty in obtaining exact solutions to many fractional differential equations, there is a large number of papers dealing with the numerical solution of fractional differential equation [16-21]. The usual spectral methods based on Legendre, Chebyshev, and Jacobi polynomials are only available for bounded domains for the numerical solution of FDEs [22, 23]. In [22, 24, 25], the authors discussed the operational matrices of Caputo fractional derivatives of shifted Chebyshev and Jacobi polynomials with spectral techniques to provide numerical approximations to the solution of linear and nonlinear multiterm FDEs on finite intervals. Ahmadian et al. [26] applied the Jacobi operational matrix to present an efficient algorithm for solving a class of fuzzy FDEs. Li et al. [27] employed spectral approximations to compute the fractional integral and the Caputo derivative; 
they also presented pseudospectral approximation for a class of FDEs. Yüzbaşı [28] proposed Bessel collocation method for the approximate solution of the Bagley-Torvik equation. Atabakzadeh et al. [29] extended the application of the shifted Chebyshev operational matrix for obtaining an analytical approximation solution for linear and nonlinear multiorder FDE. Moreover, the authors of [30] extended the application of the Legendre operational matrix for treating the fractional order fuzzy differential equations. Recently, Bhrawy et al. [31, 32] proposed the operational matrices of Riemann-Liouville fractional integration of Chebyshev and modified generalized Laguerre polynomials which are employed together with spectral tau method for solving linear FDEs on finite and semi-infinite intervals, respectively.

The fundamental aim of this paper is to extend the application of spectral tau and collocation methods based on modified generalized Laguerre polynomials to solve the linear and nonlinear multiterm fractional initial value problems on the half line. We propose the modified generalized Laguerre spectral tau (MGLT) approximation for obtaining a direct solution technique to solve linear multiterm FDE on the half line. This method requires the Caputo fractional derivatives of any fractional order of the modified generalized Laguerre polynomials of any degree which is already stated and proved.

The modified generalized Laguerre spectral collocation (MGLC) approximation, which is more reliable, is employed to obtain approximate solution of nonlinear multiterm FDE on the half line with leading fractional differential operator of order $v(m-1<v \leq m)$ and $m$ initial conditions. To be more precise, in such approximation, the nonlinear FDE is collocated only at $(N-m+1)$ zeros of modified generalized Laguerre polynomials. Therefore, the problem reduces to a system of $(N+1)$ nonlinear algebraic equations which may be solved by any standard technique to find the spectral solution $u_{N}(x)$. To the best of the our knowledge, the proposed algorithms have not been introduced for the numerical solution of multiterm FDEs on the half line.

The plan of the paper is as follows. In the next section, we introduce basic properties of modified generalized Laguerre polynomials. In Section 3, the Caputo fractional derivative of the modified generalized Laguerre polynomials is proved. In Section 4, we develop modified generalized Laguerre tau and collocation spectral methods for solving multiterm FDEs. In Section 5, several numerical examples are implemented. Also, a conclusion is given in Section 6.

\section{Properties of Modified Generalized Laguerre Polynomials}

We recall below some relevant properties of the modified generalized Laguerre polynomials (see $[8,33,34]$ ).

Now, let $\Lambda=(0, \infty)$ and $w^{(\alpha, \beta)}(x)=x^{\alpha} e^{-\beta x}$ be a weight function on $\Lambda$ in the usual sense. Define

$$
\begin{gathered}
L_{w^{(\alpha, \beta)}}^{2}(\Lambda)=\{v \mid v \text { is measurable on } \Lambda \\
\text { and } \left.\|v\|_{w^{(\alpha, \beta)}}<\infty\right\},
\end{gathered}
$$

equipped with the following inner product and norm:

$$
\begin{gathered}
(u, v)_{w^{(\alpha, \beta)}}=\int_{\Lambda} u(x) v(x) w^{(\alpha, \beta)}(x) d x \\
\|v\|_{w^{(\alpha, \beta)}}=(v, v)_{w^{(\alpha, \beta)}}^{1 / 2} .
\end{gathered}
$$

Next, let $L_{i}^{(\alpha, \beta)}(x)$ be the modified generalized Laguerre polynomial of degree $i$ for $\alpha>-1$ and $\beta>0$, that is defined by

$$
L_{i}^{(\alpha, \beta)}(x)=\frac{1}{i !} x^{-\alpha} e^{\beta x} \partial_{x}^{i}\left(x^{i+\alpha} e^{-\beta x}\right), \quad i=1,2, \ldots
$$

According to (2.3)-(2.4) of [11] for $\alpha>-1$ and $\beta>0$, we have

$$
\begin{gathered}
\partial_{x} L_{i}^{(\alpha, \beta)}(x)=-\beta L_{i-1}^{(\alpha+1, \beta)}(x), \\
L_{i+1}^{(\alpha, \beta)}(x)=\frac{1}{i+1}\left[(2 i+\alpha+1-\beta x) L_{i}^{(\alpha, \beta)}(x)\right. \\
\left.\quad-(i+\alpha) L_{i-1}^{(\alpha, \beta)}(x)\right], \quad i=1,2, \ldots,
\end{gathered}
$$

where $L_{0}^{(\alpha, \beta)}(x)=1$ and $L_{1}^{(\alpha, \beta)}(x)=-\beta x+\Gamma(\alpha+2) / \Gamma(\alpha+1)$.

The set of modified generalized Laguerre polynomials is the $L_{w^{(\alpha, \beta)}}^{2}(\Lambda)$-orthogonal system, namely,

$$
\int_{0}^{\infty} L_{j}^{(\alpha, \beta)}(x) L_{k}^{(\alpha, \beta)}(x) w^{(\alpha, \beta)}(x) d x=h_{k} \delta_{j k},
$$

where $\delta_{j k}$ is the Kronecker function and $h_{k}=\Gamma(k+\alpha+$ 1) $/ \beta^{\alpha+1} k$ !.

The modified generalized Laguerre polynomials of degree $i$ on the interval $\Lambda$, are given by

$$
\begin{array}{r}
L_{i}^{(\alpha, \beta)}(x)=\sum_{k=0}^{i}(-1)^{k} \frac{\Gamma(i+\alpha+1) \beta^{k}}{\Gamma(k+\alpha+1)(i-k) ! k !} x^{k}, \\
i=0,1, \ldots,
\end{array}
$$

where $L_{i}^{(\alpha, \beta)}(0)=\Gamma(i+\alpha+1) / \Gamma(\alpha+1) \Gamma(i+1)$.

The special value

$$
D^{q} L_{i}^{(\alpha, \beta)}(0)=\frac{(-1)^{q} \beta^{q} \Gamma(i+\alpha+1)}{(i-q) ! \Gamma(q+\alpha+1)}, \quad i \geqslant q,
$$

will be of important use later.

A function $u(x) \in L_{w^{(\alpha, \beta)}}^{2}(\Lambda)$ may be expressed in terms of modified generalized Laguerre polynomials as

$$
\begin{array}{r}
u(x)=\sum_{j=0}^{\infty} a_{j} L_{j}^{(\alpha, \beta)}(x), \\
a_{j}=\frac{1}{h_{j}} \int_{0}^{\infty} u(x) L_{j}^{(\alpha, \beta)}(x) w^{(\alpha, \beta)}(x) d x \\
j=0,1,2, \ldots
\end{array}
$$


In particular applications, the modified generalized Laguerre polynomials up to degree $N+1$ are considered. Then, we have

$$
u_{N}(x)=\sum_{j=0}^{N} a_{j} L_{j}^{(\alpha, \beta)}(x)
$$

We now turn the modified generalized Laguerre-Gausstype quadratures, including modified generalized Laguerre-Gauss and Gauss-Radau interpolations $[11,34,35]$. Let $\xi_{G, i, j}^{(\alpha, \beta)}$ and $\xi_{R, i, j}^{(\alpha, \beta)}, 0 \leq j \leq i$, be the zeros of $L_{i+1}^{(\alpha, \beta)}(x)$ and $x \partial_{x} L_{i+1}^{(\alpha, \beta)}(x)$, respectively. They are arranged in ascending order. Denote $\omega_{Z, i, j}^{(\alpha, \beta)},(0 \leq j \leq i), Z=G, R$, the corresponding Christoffel numbers such that

$$
\int_{\Lambda} \phi(x) w^{(\alpha, \beta)}(x) d x=\sum_{j=0}^{i} \phi\left(\xi_{Z, i, j}^{(\alpha, \beta)}\right) \omega_{Z, i, j}^{(\alpha, \beta)}
$$

For the modified generalized Laguerre-Gauss weights, we have

$$
\omega_{G, i, j}^{(\alpha, \beta)}=\frac{\Gamma(i+\alpha+2)}{\beta^{\alpha} \Gamma(i+2)} \frac{1}{\xi_{G, i, j}^{(\alpha, \beta)}\left[\partial_{x} L_{i+1}^{(\alpha, \beta)}\left(\xi_{G, i, j}^{(\alpha, \beta)}\right)\right]^{2}}, \quad 0 \leq j \leq i .
$$

For the Gauss-Radau weights, we have

$$
\begin{aligned}
& \omega_{R, i, j}^{(\alpha, \beta)} \\
& = \begin{cases}\frac{(\alpha+1) \Gamma^{2}(\alpha+1) \Gamma(i+1)}{\beta^{\alpha+1} \Gamma(i+\alpha+2)}, & j=0, \\
\frac{\Gamma(i+\alpha+1)}{\beta^{\alpha} \Gamma(i+2)} \frac{1}{L_{i+1}^{(\alpha, \beta)}\left(\xi_{R, i, j}^{(\alpha, \beta)}\right) \partial_{x} L_{i}^{(\alpha, \beta)}\left(\xi_{R, i, j}^{(\alpha, \beta)}\right)}, & 1 \leq j \leq i,\end{cases}
\end{aligned}
$$

Note that the earlier two types of quadratures have close relations,

$$
\begin{array}{r}
\xi_{R, i, j}^{(\alpha, \beta)}=\xi_{G, i-1, j-1}^{(\alpha+1, \beta)}, \quad \omega_{R, i, j}^{(\alpha, \beta)}=\left(\xi_{R, i, j}^{(\alpha, \beta)}\right)^{-1} \omega_{G, i-1, j-1}^{(\alpha+1, \beta)} \\
1 \leq j \leq i .
\end{array}
$$

\section{The Fractional Derivatives of $L_{i}^{(\alpha, \beta)}(x)$}

In this section, we prove the following theorem for expressing explicitly the fractional order derivatives of the modified generalized Laguerre polynomials in terms of the modified generalized Laguerre polynomials themselves.

Lemma 1. Let $L_{i}^{(\alpha, \beta)}(x)$ be a modified generalized Laguerre polynomial; then

$$
D^{\nu} L_{i}^{(\alpha, \beta)}(x)=0, \quad i=0,1, \ldots,\lceil\nu\rceil-1, \nu>0
$$

Theorem 2. The Caputo fractional derivative of order $v$ for modified generalized Laguerre polynomials is given by

$$
D^{\nu} L_{i}^{(\alpha, \beta)}(x)=\sum_{j=0}^{N} \Psi_{\nu}(i, j) L_{j}^{(\alpha, \beta)}(x), \quad i=\lceil\nu\rceil, \ldots, N
$$

where

$\Psi_{v}(i, j)$

$=\sum_{k=\lceil\nu\rceil \ell=0}^{i} \sum_{\ell}^{j} \frac{(-1)^{k+\ell} \beta^{v} j ! \Gamma(i+\alpha+1) \Gamma(k-v+\alpha+\ell+1)}{(i-k) !(j-\ell) ! \ell ! \Gamma(k-v+1) \Gamma(k+\alpha+1) \Gamma(\alpha+\ell+1)}$.

Proof. With the aid of (7), the $v$-order derivative of modified generalized Laguerre polynomials is

$$
\begin{aligned}
D^{\nu} L_{i}^{(\alpha, \beta)}(x) & \\
= & \sum_{k=0}^{i}(-1)^{k} \frac{\beta^{k} \Gamma(i+\alpha+1)}{(i-k) ! k ! \Gamma(k+\alpha+1)} D^{\nu} x^{k} \\
& =\sum_{k=\lceil\nu\rceil}^{i}(-1)^{k} \frac{\beta^{k} \Gamma(i+\alpha+1)}{(i-k) ! \Gamma(k-\nu+1) \Gamma(k+\alpha+1)} x^{k-\nu}, \\
i & =\lceil\nu\rceil, \ldots, N .
\end{aligned}
$$

The approximation of $x^{k-v}$ by $N+1$ terms of modified generalized Laguerre series yields

$$
x^{k-v}=\sum_{j=0}^{N} b_{j} L_{j}^{(\alpha, \beta)}(x)
$$

where $b_{j}$ is given by

$$
b_{j}=\sum_{\ell=0}^{j}(-1)^{\ell} \frac{\beta^{-k+v} j ! \Gamma(k-v+\alpha+\ell+1)}{(j-\ell) !(\ell) ! \Gamma(\ell+\alpha+1)},
$$

Thanks to (18)-(20), we can write

$$
D^{\nu} L_{i}^{(\alpha, \beta)}(x)=\sum_{j=0}^{N} \Psi_{\nu}(i, j) L_{j}^{(\alpha, \beta)}(x), \quad i=\lceil\nu\rceil, \ldots, N
$$

where $\Psi_{\nu}(i, j)=\sum_{k=\lceil\nu\rceil}^{i} \theta_{i j k}$, and

$\theta_{i j k}$

$=\sum_{\ell=0}^{j} \frac{(-1)^{k+\ell} \beta^{v} j ! \Gamma(i+\alpha+1) \Gamma(k-v+\alpha+\ell+1)}{(i-k) !(j-\ell) ! \ell ! \Gamma(k-v+1) \Gamma(k+\alpha+1) \Gamma(\alpha+\ell+1)}$. 


\section{Application of the Fractional Derivative of Modified Generalized Laguerre Polynomials}

In this section, we consider spectral tau and collocation methods based on the fractional derivative of modified generalized Laguerre polynomials to solve numerically the linear and nonlinear multiterm FDEs on the half line.

4.1. Tau Method for Linear Multiterm FDEs. We apply the modified generalized Laguerre tau (MGLT) method for tackling the linear multiterm FDEs on the half line

$$
D^{\nu} u(x)=\sum_{j=1}^{k} \gamma_{j} D^{\zeta_{j}} u(x)+\gamma_{k+1} u(x)+g(x), \quad \text { in } \Lambda,
$$

subject to initial conditions

$$
u^{(i)}(0)=d_{i}, \quad i=0, \ldots, m-1,
$$

where $\gamma_{j}(j=1, \ldots, k+1)$ are constant coefficients, $m-1<$ $v \leq m, 0<\zeta_{1}<\zeta_{2}<\cdots<\zeta_{k}<\nu$. While $D^{\nu} u(x) \equiv u^{(\nu)}(x)$ is the $v$-order fractional derivative of $u(x)$, and $g(x)$ is a source function.

The modified generalized Laguerre polynomials $\left\{L_{i}^{(\alpha, \beta)}(x): i \geq 0\right\}$ form a complete orthogonal system in weighted space; $L_{w^{(\alpha, \beta)}}^{2}(\Lambda)$. Hence, we define

$$
S_{N}(\Lambda)=\operatorname{span}\left\{L_{0}^{(\alpha, \beta)}(x), L_{1}^{(\alpha, \beta)}(x), \ldots, L_{N}^{(\alpha, \beta)}(x)\right\},
$$

then the standard modified generalized Laguerre tau approximation to (23) is to find $u_{N} \in S_{N}(\Lambda)$ such that

$$
\begin{aligned}
\left(D^{v} u_{N}, L_{k}^{(\alpha, \beta)}(x)\right)_{w^{(\alpha, \beta)}}+\sum_{i=1}^{r-1} \gamma_{i}\left(D^{\zeta_{i}} u_{N}, L_{k}^{(\alpha, \beta)}(x)\right)_{w^{(\alpha, \beta)}} \\
+\gamma_{r}\left(u_{N}, L_{k}^{(\alpha, \beta)}(x)\right)_{w^{(\alpha, \beta)}} \\
=\left(\left(g, L_{k}^{(\alpha, \beta)}(x)\right)_{w^{(\alpha, \beta)}}, \quad k=0,1, \ldots, N-m,\right. \\
u_{N}^{(i)}(0)=d_{i}, \quad i=0,1, \ldots, m-1 .
\end{aligned}
$$

where

Let us denote

$$
\begin{gathered}
u_{N}(x)=\sum_{j=0}^{N} a_{j} L_{j}^{(\alpha, \beta)}(x), \quad \mathbf{a}=\left(a_{0}, a_{1}, \ldots, a_{N}\right)^{T}, \\
g_{k}=\left(g, L_{k}^{(\alpha, \beta)}(x)\right)_{w^{(\alpha, \beta)}}, \quad k=0,1, \ldots, N-m, \\
\mathbf{g}=\left(g_{0}, g_{1}, \ldots, g_{N-m}, d_{0}, \ldots, d_{m-1}\right)^{T},
\end{gathered}
$$

then (26) can be written as

$$
\begin{aligned}
& \sum_{j=0}^{N} a_{j}\left[\left(D^{\nu} L_{j}^{(\alpha, \beta)}(x), L_{k}^{(\alpha, \beta)}(x)\right)_{w^{(\alpha, \beta)}}\right. \\
& \quad+\sum_{i=1}^{r-1} \gamma_{i}\left(D^{\zeta} L_{j}^{(\alpha, \beta)}(x), L_{k}^{(\alpha, \beta)}(x)\right)_{w^{(\alpha, \beta)}} \\
& \left.\quad+\gamma_{r}\left(L_{j}^{(\alpha, \beta)}(x), L_{k}^{(\alpha, \beta)}(x)\right)_{w^{(\alpha, \beta)}}\right] \\
& =\left(g, L_{k}^{(\alpha, \beta)}(x)\right)_{w^{(\alpha, \beta)}}, \quad k=0,1, \ldots, N-m, \\
& \sum_{j=0}^{N} a_{j} L_{j}^{(\alpha, \beta)(k-N+m-1)}(0) \\
& =d_{(k-N+m-1)}, \quad k=N-m+1, N-m+2, \ldots, N .
\end{aligned}
$$

Let us also denote

$$
\begin{gathered}
A=\left(a_{k j}\right)_{0<k, j<N}, \\
B^{s}=\left(b_{k j}^{s}\right)_{0<k, j<N ; s=1,2, \ldots, r-1}, \\
C=\left(c_{k j}\right)_{0<k, j<N},
\end{gathered}
$$

then by virtue of the orthogonality relation of modified generalized Laguerre polynomials (6) and making use of (8) and (16) and after some manipulation, we may deduce that the elements of $a_{k j}, b_{k j}^{s}, s=1,2, \ldots, r-1$, and $c_{k j}$ are given by 


$$
\begin{gathered}
a_{k j}= \begin{cases}h_{k} \Psi_{v}(j, k), & k=0,1, \ldots, N-m, j=m, m+1, \ldots, N, \\
\frac{(-1)^{k-N+m-1} \beta^{(k-N+m-1)} \Gamma(i+\alpha+1) !}{(i-k+N-m+1) ! \Gamma(k-N+m+\alpha+2)}, & k=N-m+1, \ldots, N, j=0,1, \ldots, N,\end{cases} \\
b_{k j}^{s}= \begin{cases}h_{k} \Psi_{\zeta_{s}}(j, k), & k=0,1, \ldots, N-m, j=\left\lceil\zeta_{s}\right\rceil,\left\lceil\zeta_{s}\right\rceil+1, \ldots, N, s=1,2, \ldots, r-1, \\
0, & \text { otherwise, }\end{cases} \\
c_{k j}= \begin{cases}h_{k}, & k=j=0,1, \ldots, N-m, \\
0, & \text { otherwise. }\end{cases}
\end{gathered}
$$

Therefore, (28) can be written in the following matrix form:

$$
\left(A+\sum_{i=1}^{r-1} \gamma_{i} B^{i}+\gamma_{r} C\right) \mathbf{a}=\mathbf{g} .
$$

4.2. Collocation Method for Nonlinear Multiterm FDEs. It is known that the collocation method approximates differential equations in physical space, so it is easy to apply to various problems, including variable coefficient and nonlinear differential equations (see, for instance, [36]). In this section, an algorithm for solving fractional differential equation is proposed based on modified generalized Laguerre spectral collocation (MGLC) method in a half line. Here, we study the following multiterm FDE:

$$
D^{v} u(x)=F\left(x, u(x), D^{\beta_{1}} u(x), \ldots, D^{\beta_{k}} u(x)\right), \quad \text { in } \Lambda,
$$

with initial conditions

$$
u^{(i)}(0)=d_{i}, \quad i=0, \ldots, m-1,
$$

where the fractional order derivatives are taken to be of the Caputo type, $m-1<v \leq m, 0<\beta_{1}<\beta_{2}<\cdots<\beta_{k}<v$, and $F$ is a nonlinear function.

We now derive an efficient algorithm for solving nonlinear multiterm FDEs (33)-(34). We expand the numerical approximation in terms of modified generalized Laguerre polynomials

$$
u_{N}(x)=\sum_{j=0}^{N} a_{j} L_{j}^{(\alpha, \beta)}(x)
$$

By virtue of (16), the fractional derivatives $D^{v} u(x)$, $D^{\beta_{1}} u(x), \ldots, D^{\beta_{k}} u(x)$ can be expressed explicitly in terms of the expansion coefficients $a_{j}$.

The modified generalized Laguerre spectral collocation method for solving approximately (33), (34) is to find $u_{N}(x) \in$ $S_{N}(\Lambda)$ such that

$$
D^{\nu} u_{N}(x)=F\left(x, u_{N}(x), D^{\beta_{1}} u_{N}(x), \ldots, D^{\beta_{k}} u_{N}(x)\right),
$$

is collocated at $x_{N-m+1, k}, k=0,1, \ldots, N-m$. In other words, we have to collocate $(36)$ at the $(N-m+1)$ modified generalized Laguerre roots $x_{N-m+1, k}$, which immediately yields

$$
\begin{aligned}
& \sum_{j=0}^{N} a_{j} D^{\nu} L_{j}^{(\alpha, \beta)}\left(x_{N-m+1, k}\right) \\
& =F\left(x_{N-m+1, k,} \sum_{j=0}^{N} a_{j} D^{\beta_{1}} L_{j}^{(\alpha, \beta)}\left(x_{N-m+1, k}\right), \ldots,\right. \\
& \left.\sum_{j=0}^{N} a_{j} D^{\beta_{k}} L_{j}^{(\alpha, \beta)}\left(x_{N-m+1, k}\right)\right) \\
& k=0,1, \ldots, N-m \text {, }
\end{aligned}
$$

with (33) written in the form

$$
\sum_{j=0}^{N} a_{j} L_{j}^{(\alpha, \beta)}(0)=d_{i}, \quad i=0,1, \ldots, m-1 .
$$

Finally, the problem is reduced to solve a system of $(N+$ 1) nonlinear algebraic equations, (37)-(38), in the unknown expansion coefficients $\left\{a_{j} ; j=0,1, \ldots, N\right\}$, which can be solved by Newton's iteration method.

\section{Numerical Results}

We report in this section some numerical results obtained with the proposed algorithms in the previous section. Several test examples are implemented to ensure the effectiveness of the proposed methods for fractional differential equations in the half line.

Example 3. This example concerns a class of Bagley-Torvik equation given by the initial value problem

$$
\begin{array}{r}
D^{2} u(x)+D^{3 / 2} u(x)+u(x)=x^{3}+6 x+\frac{8}{\Gamma(0.5)} x^{1.5}, \\
u(0)=0, \quad u^{\prime}(0)=0, \quad x \in[0,10] .
\end{array}
$$

The solution is $u(x)=x^{3}$.

This equation arises in the treatment of the motion of a large thin plate immersed in a Newtonian fluid. 


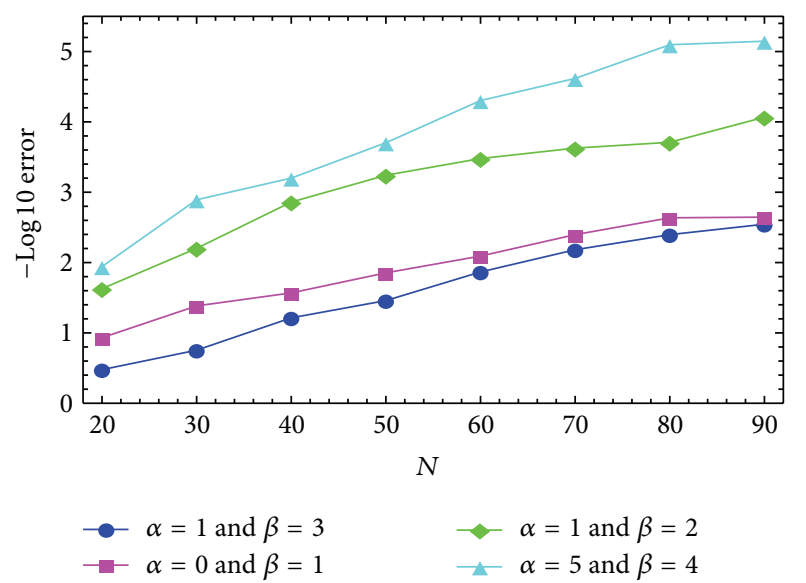

FIGURE 1: Maximum absolute error by using MGLT (Gauss) with the various choices of $N$.

For the numerical solution of the Bagley-Torvik equation, see [37-41]. The solution of this problem is obtained by applying the modified generalized Laguerre tau method and the righthand side is treated by modified generalized Laguerre-Gauss $(\mathrm{G})$ and modified generalized Laguerre-Gauss-Radau (GR) quadratures with general parameters $\alpha$ and $\beta$. The maximum absolute errors obtained by MGLT method for various choices of $N, \alpha$, and $\beta$ are given in Figures 1 and 2. Moreover, the approximate solutions obtained by the proposed method for $\alpha=0, \beta=2$, and two choices of $N$ are shown in Figure 3 to make it easier to compare with the analytic solution.

Example 4. Consider the equation

$$
\begin{aligned}
& D^{2} u(x)+D^{v} u(x)+u(x)=g(x), \\
& u(0)=0, \quad u^{\prime}(0)=0, \quad 0<v<1, \quad x \in[0,10],
\end{aligned}
$$

where

$$
\begin{aligned}
g(x)= & x^{4}-\frac{1}{2} x^{3}+12 x^{2}-3 x \\
& -\frac{3}{\Gamma(4-\nu)} x^{3-\nu}+\frac{24}{\Gamma(5-\nu)} x^{4-\nu},
\end{aligned}
$$

and the exact solution is given by $u(x)=x^{4}-(1 / 2) x^{3}$ (see Table 1).

Example 5. We next consider the following:

$$
\begin{aligned}
& D^{2} u(x)+D^{v} u(x)+u(x)=g(x), \\
& u(0)=1, \quad u^{\prime}(0)=0, \quad x \in[0,10],
\end{aligned}
$$

where

$$
g(x)=\left(1-\gamma^{2}\right) \cos (\gamma x)+\frac{1}{\Gamma(-\nu)} \int_{0}^{x}(x-t)^{-\nu-1} u(t) d t,
$$

and the exact solution is given by $u(x)=\cos (\gamma x)$.

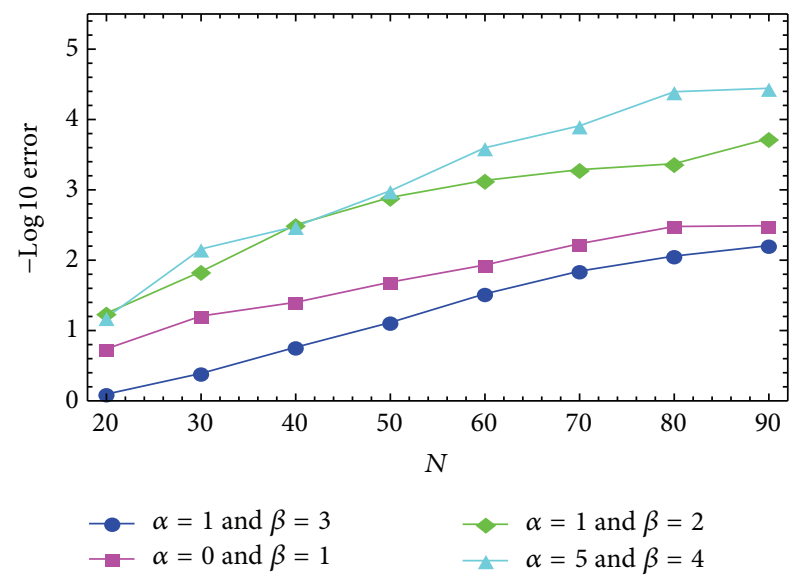

FIgure 2: Maximum absolute error by using MGLT (Radau) with the various choices of $N$.

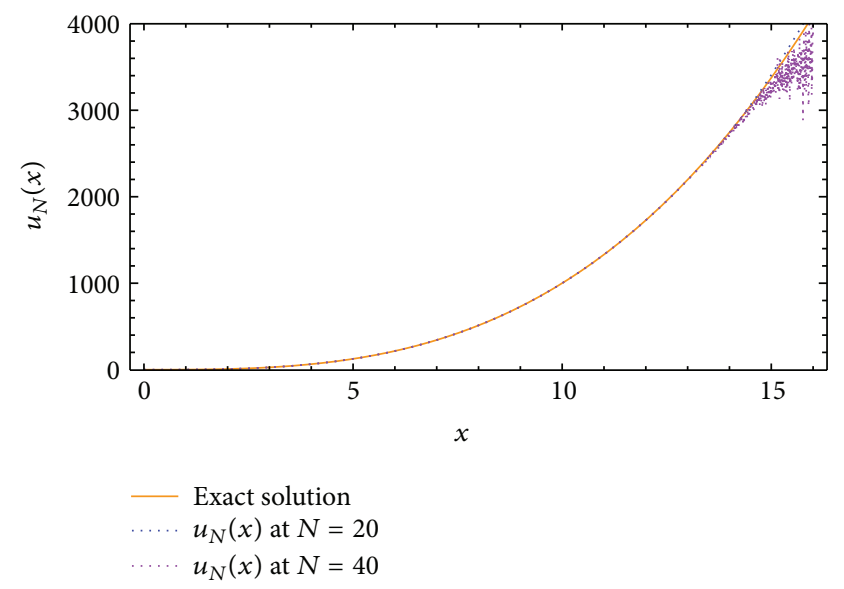

FIGURE 3: Comparing the exact and approximate solutions at $N=$ 20, 40, with $\alpha=0$ and $\beta=2$.

The solution of this problem is obtained by applying the technique described in Section 4. The maximum absolute error for $\gamma=\{0.1$ and 0.01$\}$ and various choices of $N, \alpha, \beta$, and $\nu$ are shown in Tables 2 and 3; moreover, the right-hand side is treated by modified generalized Laguerre Gauss and modified generalized Laguerre-Gauss-Radau quadratures.

Example 6. We consider the equation

$$
\begin{aligned}
& a D^{\nu} u(x)+b D^{\nu_{2}} u(x)+c D^{\nu_{1}} u(x)+e u(x)=g(x), \\
& 0<v_{1}<1, \quad 1<v_{2}<2, \quad 3<v<4, \quad x \in[0,5],
\end{aligned}
$$

$$
\begin{aligned}
g(x)= & \frac{2 b}{\Gamma\left(3-v_{2}\right)} x^{2-v_{2}}+\frac{2 c}{\Gamma\left(3-v_{1}\right)} x^{2-v_{1}} \\
& -\frac{c}{\Gamma\left(2-v_{1}\right)} x^{1-v_{1}}+e\left(x^{2}-x\right),
\end{aligned}
$$


TABLE 1: Maximum absolute error, with various choices of $N$, for Example 4 in which $x \in[0,10]$.

\begin{tabular}{|c|c|c|c|c|c|c|c|c|c|c|c|}
\hline$N$ & $\alpha$ & $\beta$ & $v$ & $\operatorname{MGLT}(\mathrm{G})$ & MGLT(GR) & $v$ & MGLT(G) & MGLT(GR) & $v$ & MGLT(G) & $\operatorname{MGLT}(\mathrm{GR})$ \\
\hline 10 & \multirow{4}{*}{0} & \multirow{4}{*}{2} & \multirow{4}{*}{0.1} & $1.32 \cdot 10^{-2}$ & $2.24 \cdot 10^{-2}$ & \multirow{4}{*}{0.5} & $8.48 \cdot 10^{-2}$ & $1.41 \cdot 10^{-1}$ & \multirow{4}{*}{0.99} & $6.54 \cdot 10^{-3}$ & $1.07 \cdot 10^{-2}$ \\
\hline 30 & & & & $1.35 \cdot 10^{-4}$ & $1.78 \cdot 10^{-4}$ & & $1.37 \cdot 10^{-3}$ & $1.82 \cdot 10^{-3}$ & & $1.84 \cdot 10^{-4}$ & $2.53 \cdot 10^{-4}$ \\
\hline 50 & & & & $1.96 \cdot 10^{-5}$ & $2.47 \cdot 10^{-5}$ & & $2.45 \cdot 10^{-4}$ & $3.14 \cdot 10^{-4}$ & & $4.30 \cdot 10^{-5}$ & $5.71 \cdot 10^{-5}$ \\
\hline 70 & & & & $3.41 \cdot 10^{-6}$ & $4.20 \cdot 10^{-6}$ & & $4.86 \cdot 10^{-5}$ & $6.10 \cdot 10^{-5}$ & & $7.03 \cdot 10^{-6}$ & $9.16 \cdot 10^{-6}$ \\
\hline 10 & \multirow{4}{*}{4} & \multirow{4}{*}{3} & \multirow{4}{*}{0.1} & $4.54 \cdot 10^{-3}$ & $1.67 \cdot 10^{-2}$ & \multirow{4}{*}{0.5} & $2.36 \cdot 10^{-2}$ & $8.98 \cdot 10^{-2}$ & \multirow{4}{*}{0.99} & $1.43 \cdot 10^{-3}$ & $5.85 \cdot 10^{-3}$ \\
\hline 30 & & & & $2.86 \cdot 10^{-6}$ & $8.06 \cdot 10^{-6}$ & & $2.73 \cdot 10^{-5}$ & $8.33 \cdot 10^{-5}$ & & $2.96 \cdot 10^{-6}$ & $1.03 \cdot 10^{-5}$ \\
\hline 50 & & & & $8.61 \cdot 10^{-8}$ & $2.37 \cdot 10^{-7}$ & & $1.22 \cdot 10^{-6}$ & $3.54 \cdot 10^{-6}$ & & $1.72 \cdot 10^{-7}$ & $5.72 \cdot 10^{-7}$ \\
\hline 70 & & & & $1.81 \cdot 10^{-8}$ & $5.12 \cdot 10^{-8}$ & & $2.10 \cdot 10^{-7}$ & $6.53 \cdot 10^{-7}$ & & $3.30 \cdot 10^{-8}$ & $1.19 \cdot 10^{-7}$ \\
\hline 10 & \multirow{4}{*}{6} & \multirow{4}{*}{4} & \multirow{4}{*}{0.1} & $4.92 \cdot 10^{-3}$ & $2.27 \cdot 10^{-2}$ & \multirow{4}{*}{0.5} & $2.77 \cdot 10^{-2}$ & $1.33 \cdot 10^{-1}$ & \multirow{4}{*}{0.99} & $8.80 \cdot 10^{-3}$ & $1.67 \cdot 10^{-3}$ \\
\hline 30 & & & & $2.83 \cdot 10^{-6}$ & $1.01 \cdot 10^{-5}$ & & $2.71 \cdot 10^{-5}$ & $1.06 \cdot 10^{-4}$ & & $3.00 \cdot 10^{-6}$ & $1.35 \cdot 10^{-5}$ \\
\hline 50 & & & & $7.88 \cdot 10^{-8}$ & $2.73 \cdot 10^{-7}$ & & $5.48 \cdot 10^{-7}$ & $2.19 \cdot 10^{-6}$ & & $6.94 \cdot 10^{-8}$ & $3.25 \cdot 10^{-7}$ \\
\hline 70 & & & & $1.49 \cdot 10^{-8}$ & $5.30 \cdot 10^{-8}$ & & $1.74 \cdot 10^{-7}$ & $6.87 \cdot 10^{-7}$ & & $2.72 \cdot 10^{-8}$ & $1.26 \cdot 10^{-7}$ \\
\hline
\end{tabular}

TABLE 2: Maximum absolute error for $\gamma=1 / 10$, with various choices of $N$, for Example 5 in which $x \in[0,10]$.

\begin{tabular}{|c|c|c|c|c|c|c|c|c|c|c|c|}
\hline$N$ & $\alpha$ & $\beta$ & $v$ & MGLT(G) & MGLT(GR) & $v$ & MGLT(G) & MGLT(GR) & $v$ & MGLT(G) & MGLT(GR) \\
\hline 10 & \multirow{4}{*}{0} & \multirow{4}{*}{1} & \multirow{4}{*}{1.5} & $7.97 \cdot 10^{-4}$ & $2.47 \cdot 10^{-3}$ & \multirow{4}{*}{0.9} & $7.28 \cdot 10^{-5}$ & $1.40 \cdot 10^{-4}$ & \multirow{4}{*}{0.5} & $1.10 \cdot 10^{-4}$ & $1.89 \cdot 10^{-4}$ \\
\hline 30 & & & & $1.38 \cdot 10^{-4}$ & $3.81 \cdot 10^{-4}$ & & $7.11 \cdot 10^{-6}$ & $1.21 \cdot 10^{-5}$ & & $7.36 \cdot 10^{-6}$ & $1.11 \cdot 10^{-5}$ \\
\hline 50 & & & & $4.02 \cdot 10^{-5}$ & $1.09 \cdot 10^{-4}$ & & $1.51 \cdot 10^{-6}$ & $2.49 \cdot 10^{-6}$ & & $1.29 \cdot 10^{-6}$ & $1.88 \cdot 10^{-6}$ \\
\hline 70 & & & & $2.51 \cdot 10^{-5}$ & $6.64 \cdot 10^{-5}$ & & $3.79 \cdot 10^{-7}$ & $6.15 \cdot 10^{-7}$ & & $5.86 \cdot 10^{-7}$ & $8.41 \cdot 10^{-7}$ \\
\hline 10 & \multirow{4}{*}{2} & \multirow{4}{*}{2} & \multirow{4}{*}{1.5} & $5.39 \cdot 10^{-4}$ & $4.44 \cdot 10^{-3}$ & \multirow{4}{*}{0.9} & $6.29 \cdot 10^{-5}$ & $2.78 \cdot 10^{-4}$ & \multirow{4}{*}{0.5} & $9.84 \cdot 10^{-5}$ & $3.54 \cdot 10^{-4}$ \\
\hline 30 & & & & $1.33 \cdot 10^{-5}$ & $9.33 \cdot 10^{-5}$ & & $8.65 \cdot 10^{-7}$ & $3.20 \cdot 10^{-6}$ & & $9.31 \cdot 10^{-7}$ & $2.77 \cdot 10^{-6}$ \\
\hline 50 & & & & $4.26 \cdot 10^{-6}$ & $3.00 \cdot 10^{-5}$ & & $1.99 \cdot 10^{-7}$ & $7.37 \cdot 10^{-7}$ & & $1.71 \cdot 10^{-7}$ & $5.06 \cdot 10^{-7}$ \\
\hline 70 & & & & $1.78 \cdot 10^{-6}$ & $1.22 \cdot 10^{-5}$ & & $7.56 \cdot 10^{-8}$ & $2.72 \cdot 10^{-7}$ & & $5.28 \cdot 10^{-8}$ & $1.52 \cdot 10^{-7}$ \\
\hline 10 & \multirow{4}{*}{5} & \multirow{4}{*}{3} & \multirow{4}{*}{1.5} & $1.19 \cdot 10^{-4}$ & $1.87 \cdot 10^{-3}$ & \multirow{4}{*}{0.9} & $1.67 \cdot 10^{-5}$ & $1.34 \cdot 10^{-4}$ & \multirow{4}{*}{0.5} & $2.93 \cdot 10^{-5}$ & $1.86 \cdot 10^{-4}$ \\
\hline 30 & & & & $2.25 \cdot 10^{-6}$ & $3.48 \cdot 10^{-5}$ & & $1.58 \cdot 10^{-7}$ & $1.09 \cdot 10^{-6}$ & & $1.76 \cdot 10^{-7}$ & $1.02 \cdot 10^{-6}$ \\
\hline 50 & & & & $1.28 \cdot 10^{-6}$ & $1.92 \cdot 10^{-5}$ & & $5.85 \cdot 10^{-8}$ & $3.33 \cdot 10^{-7}$ & & $5.97 \cdot 10^{-8}$ & $3.33 \cdot 10^{-7}$ \\
\hline 70 & & & & $8.52 \cdot 10^{-7}$ & $1.26 \cdot 10^{-5}$ & & $3.21 \cdot 10^{-8}$ & $2.30 \cdot 10^{-7}$ & & $2.81 \cdot 10^{-8}$ & $1.54 \cdot 10^{-7}$ \\
\hline
\end{tabular}

TABLE 3: Maximum absolute error for $\gamma=1 / 100$, with various choices of $N$, for Example 5 in which $x \in[0,10]$.

\begin{tabular}{|c|c|c|c|c|c|c|c|c|c|c|c|}
\hline$N$ & $\alpha$ & $\beta$ & $v$ & MGLT(G) & MGLT(GR) & $v$ & MGLT(G) & MGLT(GR) & $v$ & MGLT(G) & MGLT(GR) \\
\hline 10 & \multirow{4}{*}{0} & \multirow{4}{*}{1} & \multirow{4}{*}{1.5} & $7.97 \cdot 10^{-6}$ & $2.47 \cdot 10^{-5}$ & \multirow{4}{*}{0.9} & $7.28 \cdot 10^{-7}$ & $1.40 \cdot 10^{-6}$ & \multirow{4}{*}{0.5} & $1.10 \cdot 10^{-6}$ & $1.89 \cdot 10^{-6}$ \\
\hline 30 & & & & $1.38 \cdot 10^{-6}$ & $3.81 \cdot 10^{-6}$ & & $7.11 \cdot 10^{-8}$ & $1.21 \cdot 10^{-7}$ & & $7.39 \cdot 10^{-8}$ & $1.11 \cdot 10^{-7}$ \\
\hline 50 & & & & $4.02 \cdot 10^{-7}$ & $1.09 \cdot 10^{-6}$ & & $1.51 \cdot 10^{-8}$ & $2.49 \cdot 10^{-8}$ & & $1.29 \cdot 10^{-8}$ & $1.88 \cdot 10^{-8}$ \\
\hline 70 & & & & $2.51 \cdot 10^{-7}$ & $6.64 \cdot 10^{-7}$ & & $3.79 \cdot 10^{-9}$ & $6.15 \cdot 10^{-9}$ & & $5.86 \cdot 10^{-9}$ & $8.41 \cdot 10^{-9}$ \\
\hline 10 & \multirow{4}{*}{2} & \multirow{4}{*}{2} & \multirow{4}{*}{1.5} & $5.39 \cdot 10^{-6}$ & $4.44 \cdot 10^{-5}$ & \multirow{4}{*}{0.9} & $6.29 \cdot 10^{-7}$ & $2.78 \cdot 10^{-6}$ & \multirow{4}{*}{0.5} & $9.85 \cdot 10^{-7}$ & $3.54 \cdot 10^{-6}$ \\
\hline 30 & & & & $1.33 \cdot 10^{-7}$ & $9.33 \cdot 10^{-7}$ & & $8.65 \cdot 10^{-9}$ & $3.20 \cdot 10^{-8}$ & & $9.31 \cdot 10^{-9}$ & $2.77 \cdot 10^{-8}$ \\
\hline 50 & & & & $4.26 \cdot 10^{-8}$ & $3.00 \cdot 10^{-7}$ & & $2.00 \cdot 10^{-9}$ & $7.37 \cdot 10^{-9}$ & & $1.71 \cdot 10^{-9}$ & $5.06 \cdot 10^{-9}$ \\
\hline 70 & & & & $1.78 \cdot 10^{-8}$ & $1.22 \cdot 10^{-7}$ & & $7.56 \cdot 10^{-10}$ & $2.72 \cdot 10^{-9}$ & & $5.28 \cdot 10^{-10}$ & $1.52 \cdot 10^{-9}$ \\
\hline 10 & \multirow{4}{*}{5} & \multirow{4}{*}{3} & \multirow{4}{*}{1.5} & $1.19 \cdot 10^{-6}$ & $1.87 \cdot 10^{-5}$ & \multirow{4}{*}{0.9} & $1.67 \cdot 10^{-7}$ & $1.34 \cdot 10^{-6}$ & \multirow{4}{*}{0.5} & $2.94 \cdot 10^{-7}$ & $1.87 \cdot 10^{-6}$ \\
\hline 30 & & & & $2.25 \cdot 10^{-8}$ & $3.48 \cdot 10^{-7}$ & & $1.58 \cdot 10^{-9}$ & $1.09 \cdot 10^{-8}$ & & $1.76 \cdot 10^{-9}$ & $1.02 \cdot 10^{-8}$ \\
\hline 50 & & & & $1.28 \cdot 10^{-8}$ & $1.92 \cdot 10^{-7}$ & & $5.85 \cdot 10^{-10}$ & $4.27 \cdot 10^{-9}$ & & $5.97 \cdot 10^{-10}$ & $3.33 \cdot 10^{-9}$ \\
\hline 70 & & & & $8.52 \cdot 10^{-9}$ & $1.26 \cdot 10^{-7}$ & & $3.21 \cdot 10^{-10}$ & $2.30 \cdot 10^{-9}$ & & $2.81 \cdot 10^{-10}$ & $1.53 \cdot 10^{-9}$ \\
\hline
\end{tabular}

subject to

$$
\begin{array}{ll}
u(0)=0, & u^{\prime}(0)=-1, \\
u^{\prime \prime}(0)=2, & u^{\prime \prime \prime}(0)=0 .
\end{array}
$$

The analytic solution of this problem is $u(x)=x^{2}-x$, we study two different cases of $a, b, c, e, k, \nu_{1}, v_{2}$, and $\nu$.
(I) Regarding $a=1, b=1, c=1, e=1, v_{1}=0.77$, $v_{2}=1.44$, and $v=3.91$, Figures 4 and 5 display the maximum absolute errors using MGLT method with the treatment of right-hand side by modified generalized Laguerre-Gauss and Gauss-Radau, respectively.

(II) Regarding $a=1, b=1, c=0.5, e=0.5, v_{1}=\sqrt{2} / 20$, $v_{2}=\sqrt{2}$, and $v=\sqrt{11}$, Figures 6 and 7 display 


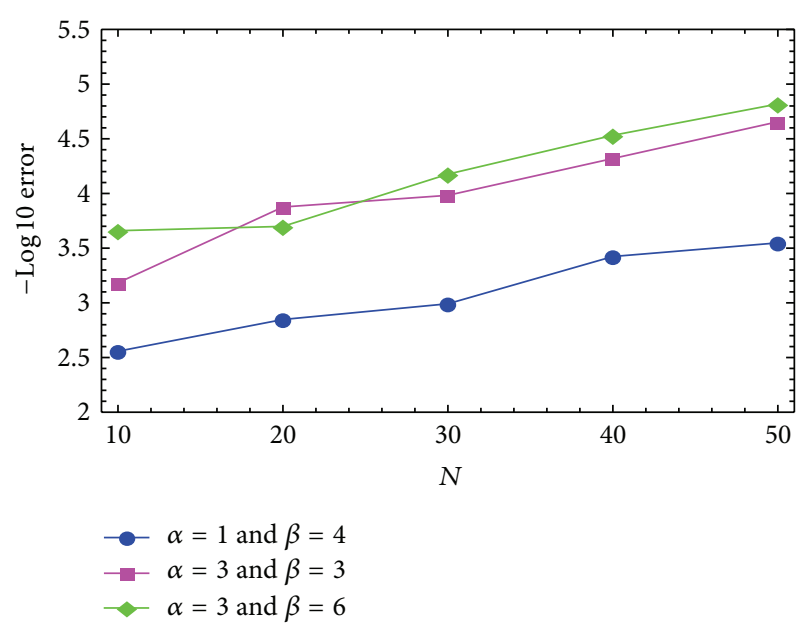

FIgURE 4: Maximum absolute error (case I) by using MGLT (Gauss) with the various choices of $N$.

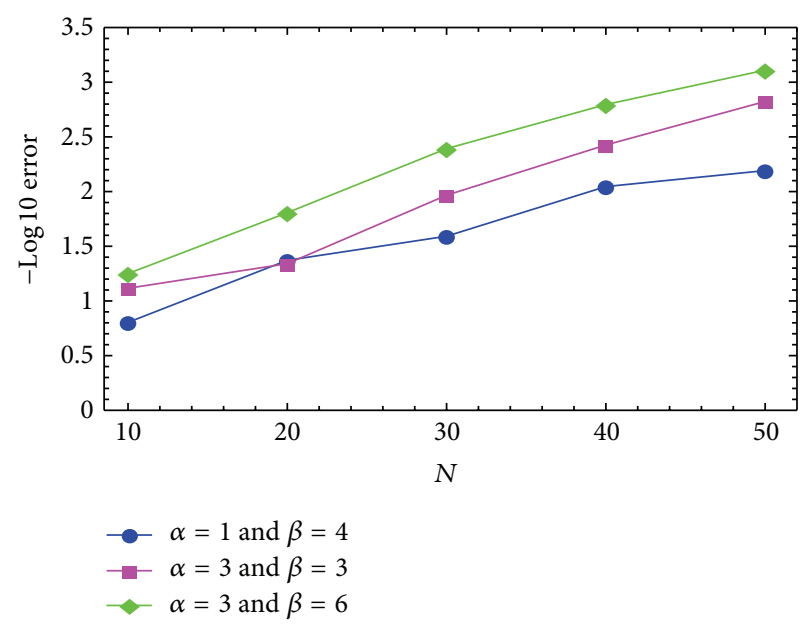

FIGURE 5: Maximum absolute error (case I) by using MGLT (Radau) with the various choices of $N$.

the maximum absolute errors using MGLT method with the treatment of right-hand side by modified generalized Laguerre-Gauss and Gauss-Radau, respectively.

Example 7. Consider the Bagley-Torvik equation with boundary conditions

$$
\begin{aligned}
& a D^{2} u(x)+b D^{v} u(x)+c u(x)=g(x), \\
& u(0)=0, \quad u(1)=0, \quad x \in(0,1),
\end{aligned}
$$

where

$$
\begin{aligned}
g(x)= & a\left(20 x^{3}-\frac{174 x^{2}}{5}+\frac{456 x}{5}-\frac{339}{125}\right) \\
& +b\left(\frac{120 x^{5-\nu}}{\Gamma(6-\nu)}-\frac{696 x^{4-\nu}}{10 \Gamma(5-\nu)}+\frac{456 x^{3-\nu}}{25 \Gamma(4-\nu)}\right.
\end{aligned}
$$

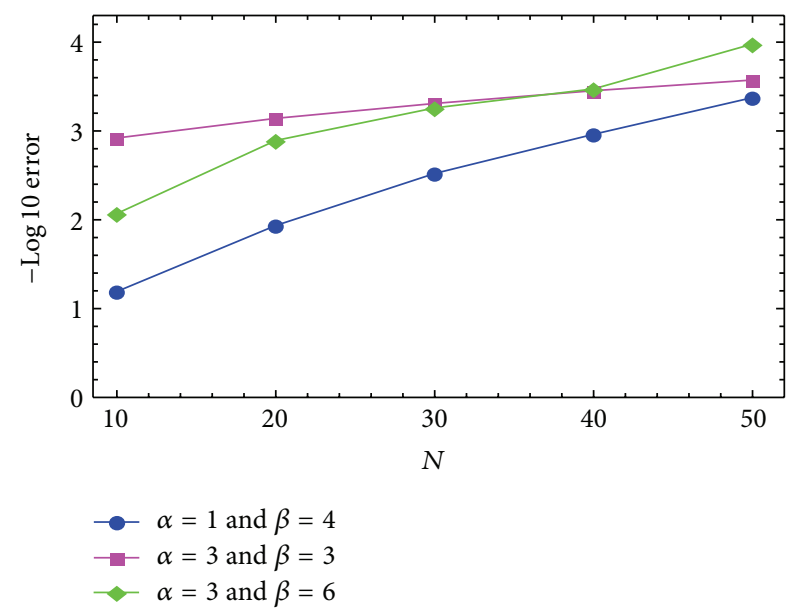

FIgURE 6: Maximum absolute error (case II) by using MGLT (Gauss) with various choices of $N$.

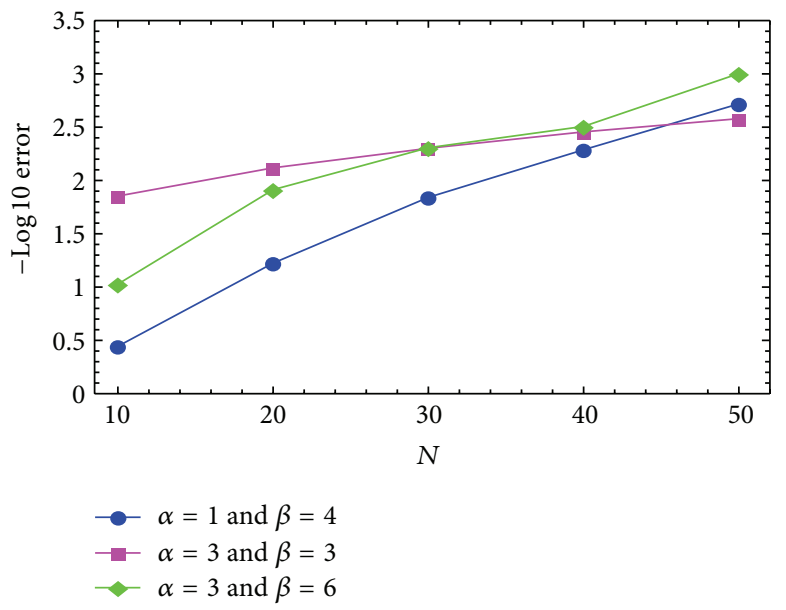

FIgure 7: Maximum absolute error (case II) by using MGLT (Radau) with various choices of $N$.

$$
\begin{gathered}
\left.-\frac{339 \Gamma(3)}{250 \Gamma(3-\nu)}\right) \\
+c\left(x^{5}-\frac{29 x^{4}}{10}+\frac{76 x^{3}}{25}-\frac{339 x^{2}}{250}+\frac{27 x}{125}\right),
\end{gathered}
$$

and the exact solution is given by $u(x)=x^{5}-\left(29 x^{4} / 10\right)+$ $\left(76 x^{3} / 25\right)-\left(339 x^{2} / 250\right)+(27 x / 125)$.

Bagley-Torvik equation involving fractional derivative of order $3 / 2$ arises in the modeling of the motion of a rigid plate in a Newtonian fluid and a gas in a fluid. We solve this equation with two-point boundary conditions where $a=1$, $b=8 / 17$, and $c=13 / 51$ by using modified generalized Laguerre-Gauss and Gauss-Radau quadratures with general parameters $\alpha$ and $\beta$. The maximum absolute errors obtained by MGLT method for various choices of $N, \alpha$, and $\beta$ are given in Table 4. Moreover, the numerical and exact solutions 
TABLE 4: Maximum absolute error for $\nu=3 / 2$, with various choices of $N$, for Example 5 in which $x \in(0,1)$.

\begin{tabular}{|c|c|c|c|c|c|c|c|c|}
\hline$N$ & $\alpha$ & $\beta$ & MGLT(G) & MGLT(GR) & $\alpha$ & $\beta$ & MGLT(G) & MGLT(GR) \\
\hline 8 & \multirow{4}{*}{0} & \multirow{4}{*}{1} & $1.69 \cdot 10^{-3}$ & $3.14 \cdot 10^{-3}$ & \multirow{4}{*}{3} & \multirow{4}{*}{4} & $8.98 \cdot 10^{-5}$ & $8.21 \cdot 10^{-4}$ \\
\hline 16 & & & $9.97 \cdot 10^{-4}$ & $2.35 \cdot 10^{-3}$ & & & $1.98 \cdot 10^{-5}$ & $1.91 \cdot 10^{-4}$ \\
\hline 32 & & & $1.57 \cdot 10^{-4}$ & $3.50 \cdot 10^{-4}$ & & & $8.50 \cdot 10^{-6}$ & $7.89 \cdot 10^{-5}$ \\
\hline 64 & & & $3.52 \cdot 10^{-5}$ & $9.97 \cdot 10^{-5}$ & & & $3.81 \cdot 10^{-6}$ & $3.72 \cdot 10^{-5}$ \\
\hline 8 & \multirow{4}{*}{4} & \multirow{4}{*}{5} & $6.47 \cdot 10^{-5}$ & $7.07 \cdot 10^{-4}$ & \multirow{4}{*}{5} & \multirow{4}{*}{5} & $8.61 \cdot 10^{-5}$ & $1.02 \cdot 10^{-3}$ \\
\hline 16 & & & $1.53 \cdot 10^{-5}$ & $1.76 \cdot 10^{-4}$ & & & $2.07 \cdot 10^{-5}$ & $2.63 \cdot 10^{-4}$ \\
\hline 32 & & & $6.40 \cdot 10^{-6}$ & $7.31 \cdot 10^{-5}$ & & & $6.17 \cdot 10^{-6}$ & $8.06 \cdot 10^{-5}$ \\
\hline 64 & & & $3.02 \cdot 10^{-6}$ & $3.57 \cdot 10^{-5}$ & & & $3.19 \cdot 10^{-6}$ & $4.37 \cdot 10^{-5}$ \\
\hline
\end{tabular}

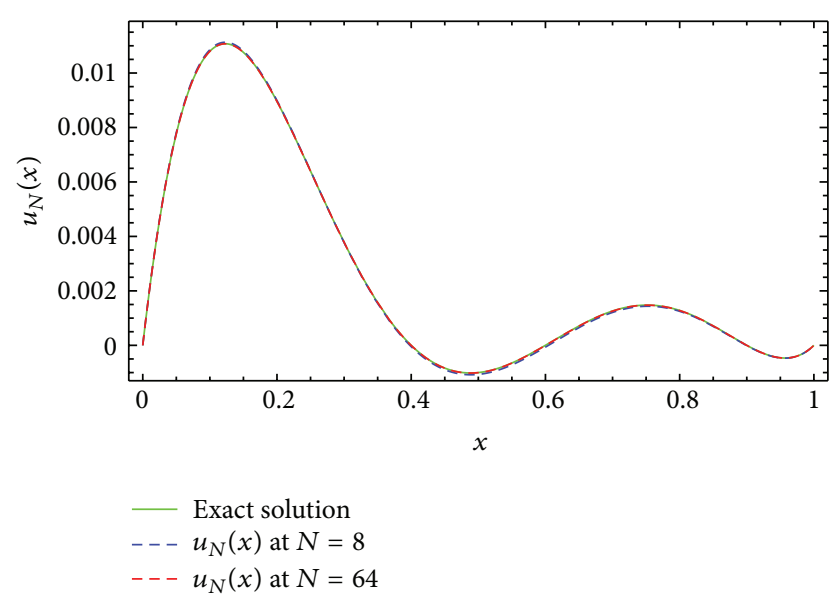

FIGURE 8: Comparing the exact and approximate solutions by using MGLT (Gauss) at $N=8,64$, with $\alpha=2$ and $\beta=4$.

obtained by the proposed method for $\alpha=2, \beta=4$, and two choices of $N$ are shown in Figures 8-9 to make it easier to compare with the analytic solution.

Example 8. We next consider the following nonlinear initial value problem:

$$
\begin{aligned}
& D^{2} u(x)+D^{3 / 4} u(x)+u^{2}(x)=g(x), \\
& u(0)=1, \quad u^{\prime}(0)=0, \quad x \in(0,10),
\end{aligned}
$$

where

$$
\begin{aligned}
g(x)= & \cos ^{2}(\gamma x)-\gamma^{2} \cos (\gamma x) \\
& +\frac{1}{\Gamma(-3 / 4)} \int_{0}^{x}(x-t)^{(-3 / 4)-1} \cos (\gamma t) d t
\end{aligned}
$$

and the exact solution is given by $u(x)=\cos (\gamma x)$.

The solution of this problem is obtained by applying the modified generalized Laguerre collocation method. The approximate solution obtained by the proposed method for $\gamma=0.01, \alpha=2, \beta=2$, and two choices of $N$ is shown in Figure 10 to make it easier to compare with the analytic solution. Moreover, the absolute error for $\gamma=0.01, \alpha=2$, $\beta=2$, and $N=20$ is given in Figure 11 .

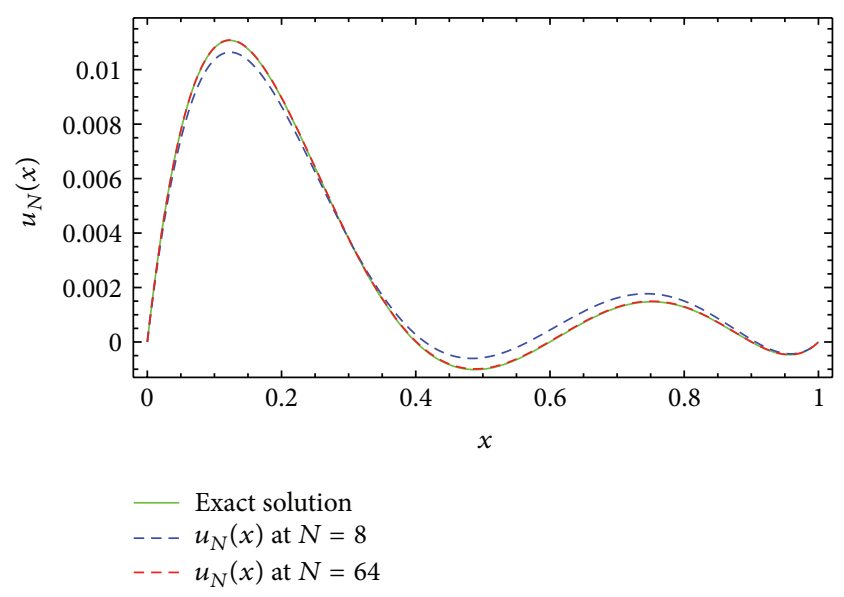

FIgURE 9: Comparing the exact and approximate solutions by using MGLT (Radau) at $N=8,64$, with $\alpha=2$ and $\beta=4$.

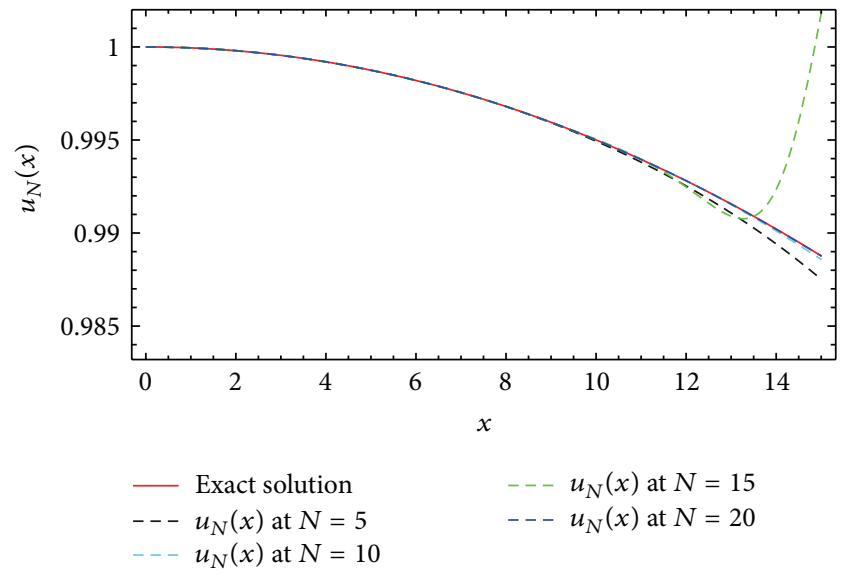

FIGURE 10: Comparing the exact and approximate solutions at $N=$ $5,10,15$, and 20, where $\nu=3 / 4, \alpha=2$, and $\beta=2$.

Example 9. Consider the following nonlinear initial value problem:

$$
D^{v} u(x)+u^{2}(x)=x+\left(\frac{x^{v+1}}{\Gamma(v+2)}\right)^{2}, \quad 0<v \leq 2,
$$

whose exact solution is given by $u(x)=(1 / \Gamma(\nu+2)) x^{\nu+1}$. 
TABLE 5: Maximum absolute error for $\nu=3 / 2$, with various choices of $N$, for Example 9 in which $x \in(0,1)$.

\begin{tabular}{|c|c|c|c|c|c|c|}
\hline$N$ & $\alpha$ & $\beta$ & MGLC & $\alpha$ & $\beta$ & MGLC \\
\hline 3 & \multirow{4}{*}{0} & \multirow{4}{*}{1} & $2.66 \cdot 10^{-2}$ & \multirow{4}{*}{2} & \multirow{4}{*}{3} & $2.61 \cdot 10^{-2}$ \\
\hline 6 & & & $2.27 \cdot 10^{-3}$ & & & $3.04 \cdot 10^{-3}$ \\
\hline 9 & & & $1.30 \cdot 10^{-3}$ & & & $1.36 \cdot 10^{-3}$ \\
\hline 12 & & & $5.68 \cdot 10^{-4}$ & & & $7.52 \cdot 10^{-4}$ \\
\hline 3 & \multirow{4}{*}{2} & \multirow{4}{*}{4} & $1.39 \cdot 10^{-2}$ & \multirow{4}{*}{3} & \multirow{4}{*}{6} & $1.07 \cdot 10^{-2}$ \\
\hline 6 & & & $1.87 \cdot 10^{-3}$ & & & $1.86 \cdot 10^{-3}$ \\
\hline 9 & & & $8.47 \cdot 10^{-4}$ & & & $6.94 \cdot 10^{-4}$ \\
\hline 12 & & & $4.02 \cdot 10^{-4}$ & & & $3.63 \cdot 10^{-4}$ \\
\hline
\end{tabular}

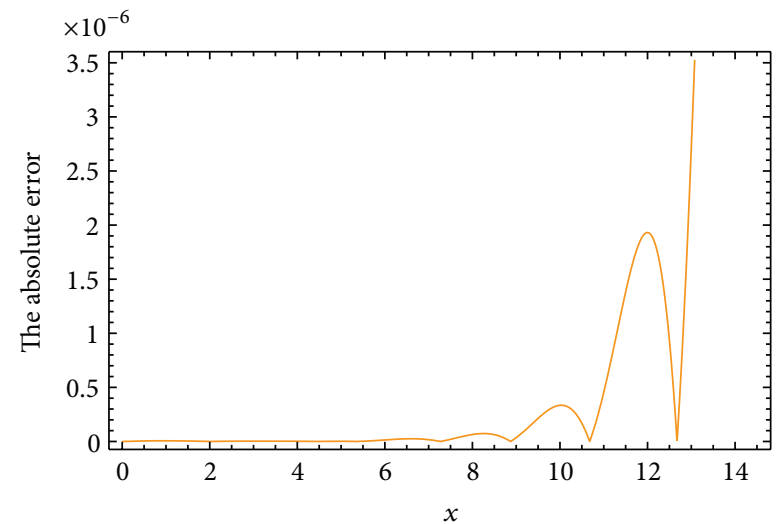

FIgure 11: The absolute error for $\gamma=1 / 100, v=3 / 4, \alpha=2$, and $\beta=2$ at $N=20$.

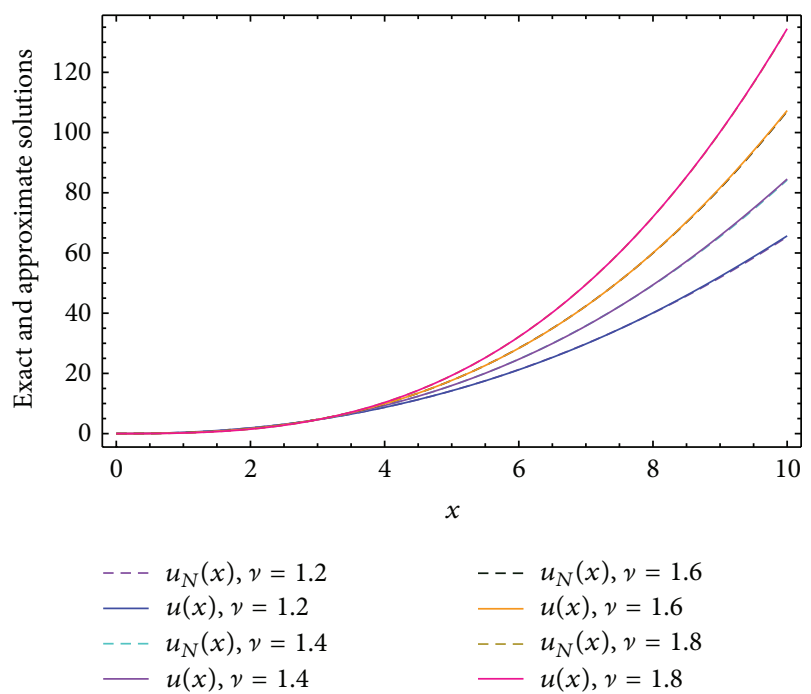

FIGURE 12: Comparing the exact and approximate solutions at $N=$ $12, \alpha=0, \beta=1$, and $\nu=1.2,1.4,1.6$, and 1.8.

Comparison between the curves of exact solutions and the approximate solutions at $\alpha=0$ and $\beta=1$ of proposed problem subject to $u(0)=u^{\prime}(0)=0$ for the four different fractional orders $v=1.2,1.4,1.6$, and 1.8 in case of $N=12$ and $N=16$ are shown in Figures 12 and 13, respectively.

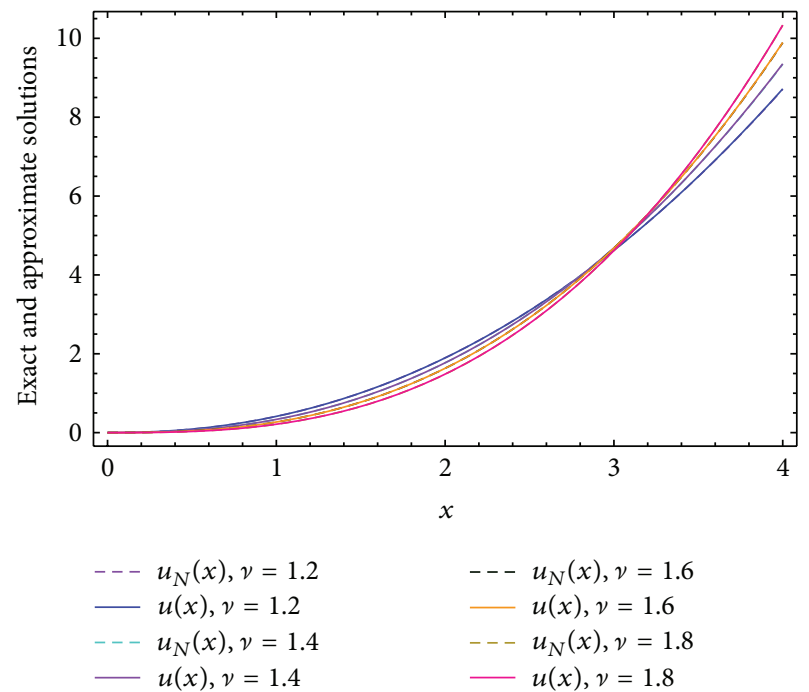

FIGURE 13: Comparing the exact and approximate solutions at $N=$ $16, \alpha=0, \beta=1$, and $\nu=1.2,1.4,1.6$, and 1.8.

The maximum absolute errors at $v=1.5$ for various choices of $\alpha, \beta$, and $N$ in the interval $[0,1]$ are shown in Table 5 . Moreover, Figures 14 and 15 display a comparison between the curves of exact solutions and the approximate solutions at $\alpha=0$ and $\beta=1$ of proposed problem subject to $u(0)=0$ for the four different fractional orders $v=0.2,0.4,0.6$, and 0.8 in case of $N=12$ and $N=16$, respectively. From all the Figures 12-15, it can be seen that the numerical solutions are in complete agreement with the exact solutions for all values of $\nu$. Also, from the numerical results implemented in this example, the classical Laguerre polynomial $(\alpha=0$, $\beta=1$ ), which is used most frequently in practice, is not the best one, especially when we are approximating the solution of fractional differential equations.

\section{Conclusions}

In this paper, we have proposed two efficient spectral methods based on modified generalized Laguerre polynomials for tackling linear and nonlinear FDEs on the half line. In these methods, the problem is reduced to the solution of a system of algebraic equations in the expansion coefficient of the solution. Numerical examples were given to demonstrate the 


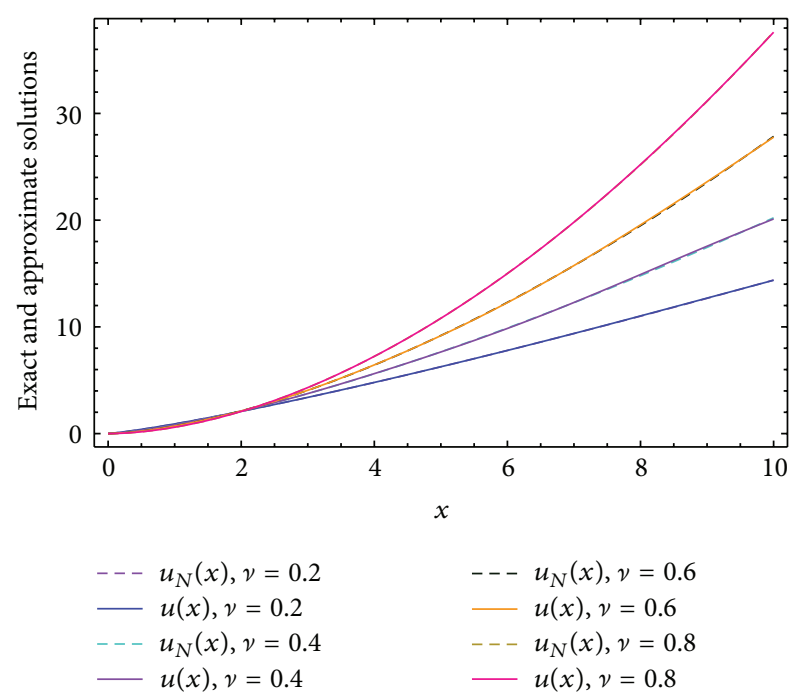

FIgURE 14: Comparing the exact and approximate solutions at $N=$ $12, \alpha=0, \beta=1$, and $\nu=0.2,0.4,0.6$, and 0.8 .

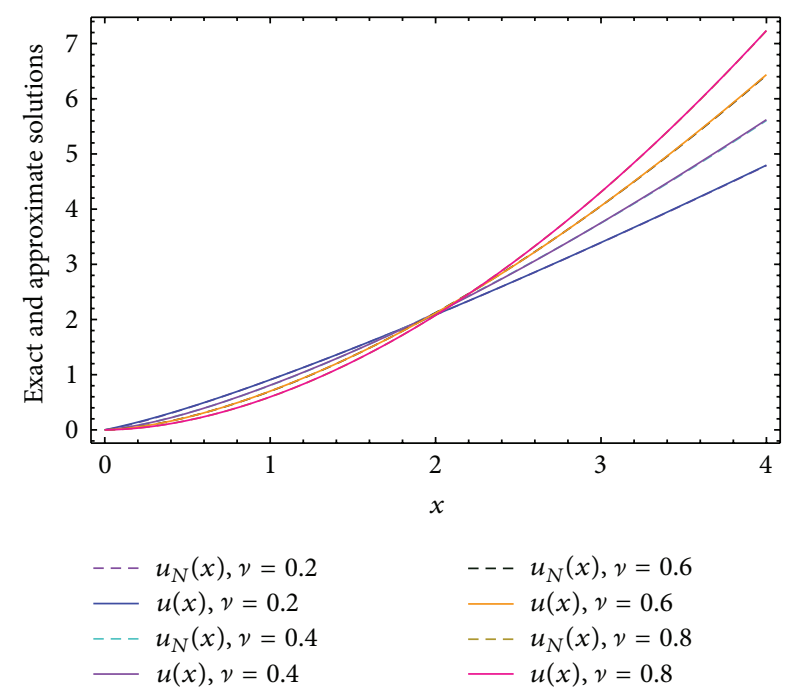

FIGURE 15: Comparing the exact and approximate solutions at $N=$ $16, \alpha=0, \beta=1$, and $\nu=0.2,0.4,0.6$, and 0.8 .

validity and applicability of the method. The computational results show that the proposed methods can be effectively used in numerical solution of time-dependent fractional partial differential equation and other problems on the half line.

\section{References}

[1] D. Baleanu, K. Diethelm, E. Scalas, and J. J. Trujillo, Fractional Calculus Models and Numerical Methods, Complexity, Nonlinearity and Chaos, World Scientific, Singapore, 2012.

[2] K. Miller and B. Ross, An Introduction to the Fractional Calaulus and Fractional Differential Equations, John Wiley \& Sons, New York, NY, USA, 1993.
[3] M. Ortigueira, "Introduction to fraction linear systems. Part 1: continuous-time case," in IEE Proceedings Vision, Image, Signal Processing, vol. 147, pp. 6-70, 2000.

[4] K. Oldham and J. Spanier, The Fractional Calculus, Academic Press, New York, NY, USA, 1974.

[5] A. A. Kilbas, H. M. Srivastava, and J. J. Trujillo, Theory and Applications of Fractional Differential Equations, vol. 204, Elsevier, Amsterdam, The Netherlands, 2006.

[6] C. Canuto, M. Y. Hussaini, A. Quarteroni, and T. A. Zang, Spectral Methods Fundamentals in Single Domains, Springer, Berlin, Germany, 2006.

[7] E. H. Doha, A. H. Bhrawy, D. Baleanu, and S. S. Ezz-Eldien, "On shifted Jacobi spectral approximations for solving fractional differential equations," Applied Mathematics and Computation, vol. 219, no. 15, pp. 8042-8056, 2013.

[8] J.-P. Yan and B.-Y. Guo, "A collocation method for initial value problems of second-order ODEs by using Laguerre functions," Numerical Mathematics. Theory, Methods and Applications, vol. 4, no. 2, pp. 283-295, 2011.

[9] E. H. Doha and A. H. Bhrawy, "An efficient direct solver for multidimensional elliptic Robin boundary value problems using a Legendre spectral-Galerkin method," Computers \& Mathematics with Applications, vol. 64, no. 4, pp. 558-571, 2012.

[10] D. Funaro, "Estimates of Laguerre spectral projectors in Sobolev spaces," in Orthogonal Polynomials and Their Applications, C. Brezinski, L. Gori, and A. Ronveaux, Eds., pp. 263-266, Scientific Publishing, Singapore, 1991.

[11] B.-Y. Guo, L.-L. Wang, and Z.-Q. Wang, "Generalized Laguerre interpolation and pseudospectral method for unbounded domains," SIAM Journal on Numerical Analysis, vol. 43, no. 6, pp. 2567-2589, 2006.

[12] M. Gülsu, B. Gürbüz, Y. Öztürk, and M. Sezer, "Laguerre polynomial approach for solving linear delay difference equations," Applied Mathematics and Computation, vol. 217, no. 15, pp. 6765-6776, 2011.

[13] R. Schimming and S. Z. Rida, "Noncommutative Bell polynomials," International Journal of Algebra and Computation, vol. 6, no. 5, pp. 635-644, 1996.

[14] A. M. A. El-Sayed and S. Z. Rida, "Bell polynomials of arbitrary (fractional) orders+," Applied Mathematics and Computation, vol. 106, no. 1, pp. 51-62, 1999.

[15] R. A. Van Gorder, "Computation of certain infinite series of the form $\Sigma f(n) n^{k}$ for arbitrary real-valued $k$," Applied Mathematics and Computation, vol. 215, no. 3, pp. 1209-1216, 2009.

[16] B. Shahriyar, F. Ismail, S. Aghabeigi, A. Ahmadian, and S. Salahshour, "An eigenvalueeigenvector method for solving a system of fractional differential equations with uncertainty," Mathematical Problems in Engineering, vol. 2013, Article ID 579761, 11 pages, 2013.

[17] A. H. Bhrawy, M. M. Tharwat, and Y. Yildirim, "A new formula for fractional integrals of Chebyshev polynomials: application for solving multi-term fractional differential equations," Applied Mathematical Modelling, vol. 37, no. 6, pp. 4245-4252, 2012.

[18] Y.-L. Jiang and X.-L. Ding, "Waveform relaxation methods for fractional differential equations with the Caputo derivatives," Journal of Computational and Applied Mathematics, vol. 238, pp. 51-67, 2013.

[19] S. Kazem, S. Abbasbandy, and S. Kumar, "Fractional-order Legendre functions for solving fractional-order differential equations," Applied Mathematical Modelling. Simulation and Computation for Engineering and Environmental Systems, vol. 37, no. 7, pp. 5498-5510, 2013. 
[20] J. Song, F. Yin, X. Cao, and F. Lu, "Fractional variational iteration method versus Adomian's decomposition method in some fractional partial differential equations," Journal of Applied Mathematics, vol. 2013, Article ID 392567, 10 pages, 2013.

[21] X. Zhang, L. Liu, Y. Wu, and Y. Lu, "The iterative solutions of nonlinear fractional differential equations," Applied Mathematics and Computation, vol. 219, no. 9, pp. 4680-4691, 2013.

[22] A. H. Bhrawy, A. S. Alofi, and S. S. Ezz-Eldien, "A quadrature tau method for fractional differential equations with variable coefficients," Applied Mathematics Letters, vol. 24, no. 12, pp. 2146-2152, 2011.

[23] E. H. Doha, A. H. Bhrawy, and S. S. Ezz-Eldien, "Efficient Chebyshev spectral methods for solving multi-term fractional orders differential equations," Applied Mathematical Modelling, vol. 35, no. 12, pp. 5662-5672, 2011.

[24] E. H. Doha, A. H. Bhrawy, and S. S. Ezz-Eldien, "A Chebyshev spectral method based on operational matrix for initial and boundary value problems of fractional order," Computers \& Mathematics with Applications, vol. 62, no. 5, pp. 2364-2373, 2011.

[25] E. H. Doha, A. H. Bhrawy, and S. S. Ezz-Eldien, "A new Jacobi operational matrix: an application for solving fractional differential equations," Applied Mathematical Modelling, vol. 36, no. 10, pp. 4931-4943, 2012.

[26] A. Ahmadian, M. Suleiman, S. Salahshour, and D. Baleanu, "A Jacobi operational matrix for solving a fuzzy linear fractional differential equation," Advances in Difference Equations, 2013, article 104.

[27] C. Li, F. Zeng, and F. Liu, "Spectral approximations to the fractional integral and derivative," Fractional Calculus and Applied Analysis, vol. 15, no. 3, pp. 383-406, 2012.

[28] S. Yüzbaşı, "Numerical solution of the Bagley-Torvik equation by the Bessel collocation method," Mathematical Methods in the Applied Sciences, vol. 36, no. 3, pp. 300-312, 2013.

[29] M. H. Atabakzadeh, M. H. Akrami, and G. H. Erjaee, "Chebyshev operational matrix method for solving multi-order fractional ordinary differential equations," Applied Mathematical Modelling, 2013.

[30] A. Ahmadian, M. Suleiman, and S. Salahshour, "An operational matrix based on Legendre polynomials for solving fuzzy fractional-order differential equations," Abstract and Applied Analysis, vol. 2013, Article ID 505903, 29 pages, 2013.

[31] A. H. Bhrawy and A. S. Alofi, "The operational matrix of fractional integration for shifted Chebyshev polynomials," Applied Mathematics Letters, vol. 26, no. 1, pp. 25-31, 2013.

[32] A. H. Bhrawy, M. Alghamdi, and T. M. Taha, "A new modified generalized Laguerre operational matrix of fractional integration for solving fractional differential equations on the half line," Advances in Difference Equations, vol. 2012, p. 179, 2012.

[33] B.-Y. Guo and X.-Y. Zhang, "A new generalized Laguerre spectral approximation and its applications," Journal of Computational and Applied Mathematics, vol. 181, no. 2, pp. 342-363, 2005.

[34] G. Szegö, Orthogonal Polynomials, vol. 23, American Mathematical Society. Colloquium Publications, 1985.

[35] B.-Y. Guo and C. Zhang, "The spectral method for high order problems with proper simulations of asymptotic behaviors at infinity," Journal of Computational and Applied Mathematics, vol. 237, no. 1, pp. 269-294, 2013.

[36] A. H. Bhrawy and A. S. Alofi, "A Jacobi-Gauss collocation method for solving nonlinear Lane-Emden type equations,"
Communications in Nonlinear Science and Numerical Simulation, vol. 17, no. 1, pp. 62-70, 2012.

[37] K. Diethelm and N. J. Ford, "Numerical solution of the BagleyTorvik equation," BIT. Numerical Mathematics, vol. 42, no. 3, pp. 490-507, 2002.

[38] S. S. Ray and R. K. Bera, "Analytical solution of the Bagley Torvik equation by Adomian decomposition method," Applied Mathematics and Computation, vol. 168, no. 1, pp. 398-410, 2005.

[39] Y. Çenesiz, Y. Keskin, and A. Kurnaz, "The solution of the Bagley-Torvik equation with the generalized Taylor collocation method," Journal of the Franklin Institute, vol. 347, no. 2, pp. $452-$ 466, 2010.

[40] M. A. Z. Raja, J. A. Khan, and I. M. Qureshi, "Solution of fractional order system of Bagley-Torvik equation using evolutionary computational intelligence," Mathematical Problems in Engineering, vol. 2011, Article ID 675075, 18 pages, 2011.

[41] S. S. Ray, “On Haar wavelet operational matrix of general order and its application for the numerical solution of fractional Bagley Torvik equation," Applied Mathematics and Computation, vol. 218, no. 9, pp. 5239-5248, 2012. 


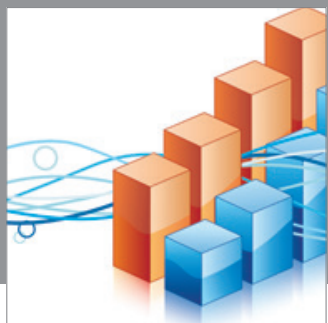

Advances in

Operations Research

mansans

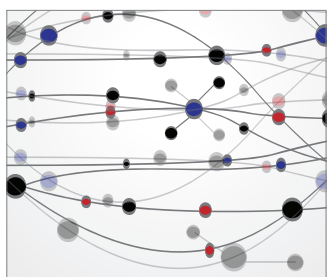

The Scientific World Journal
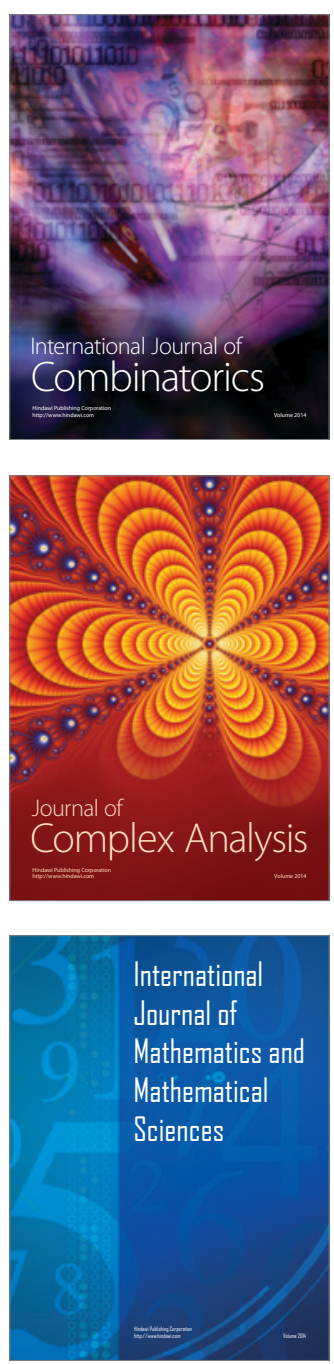
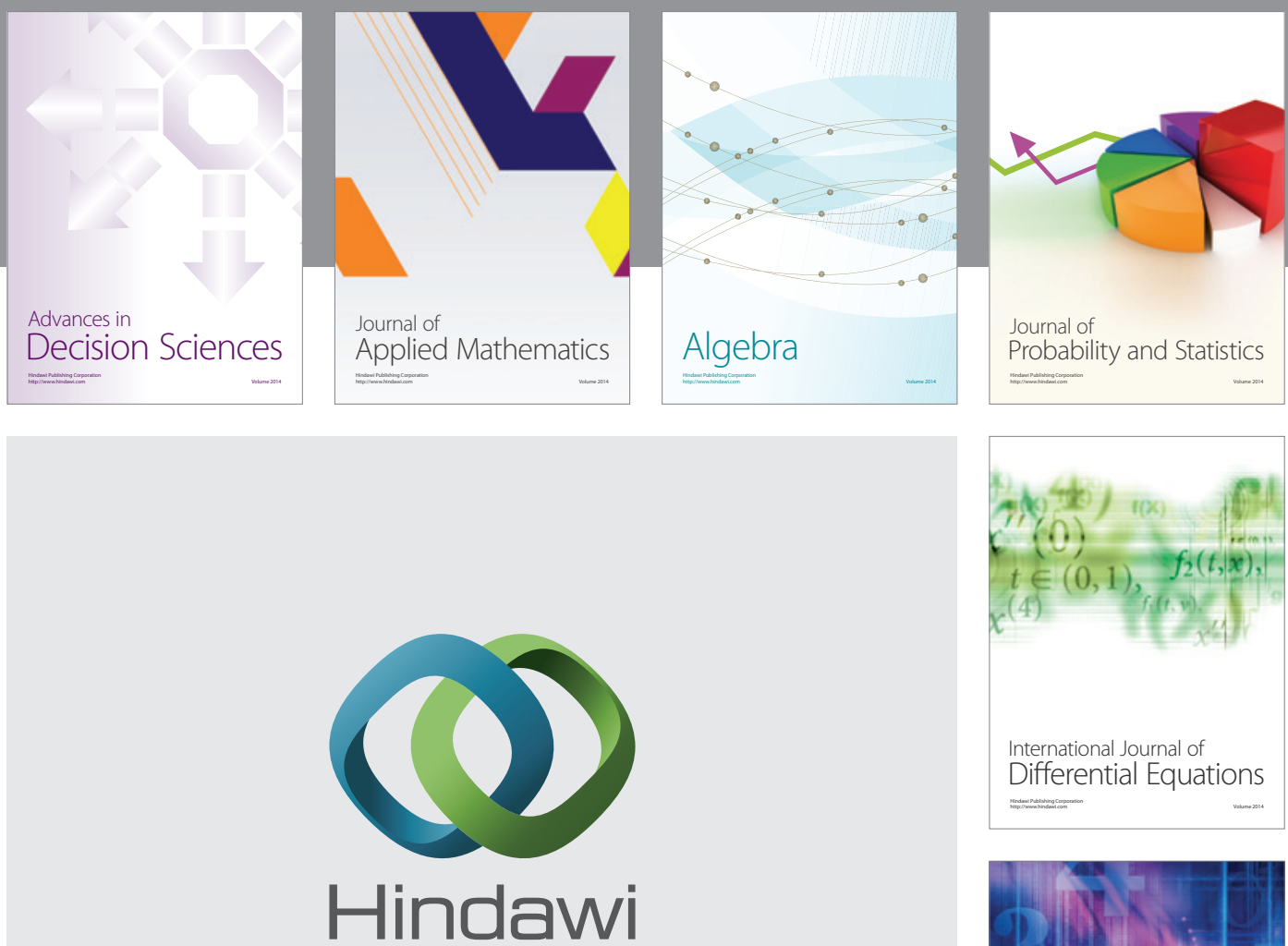

Submit your manuscripts at http://www.hindawi.com
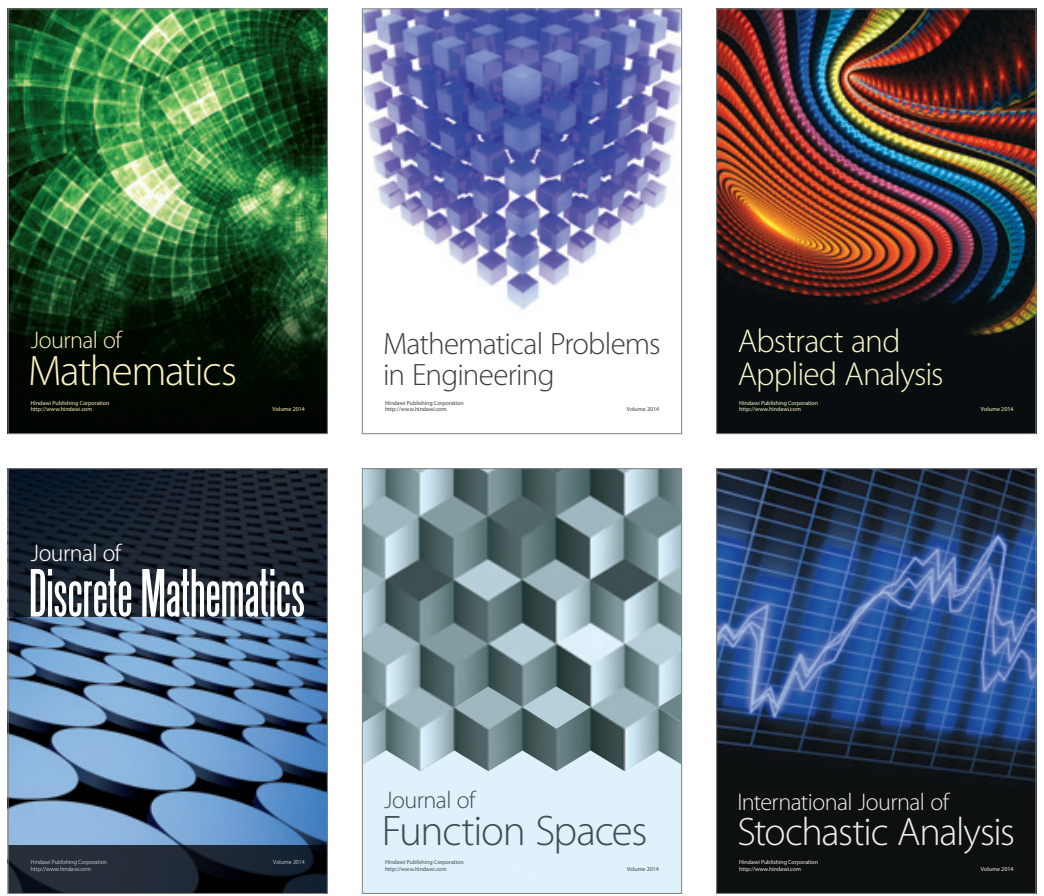

Journal of

Function Spaces

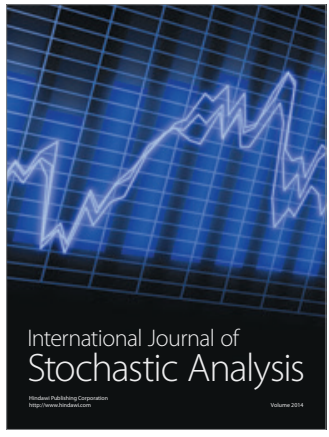

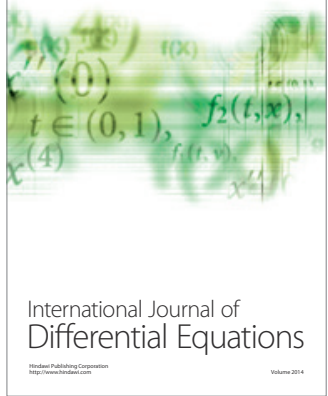
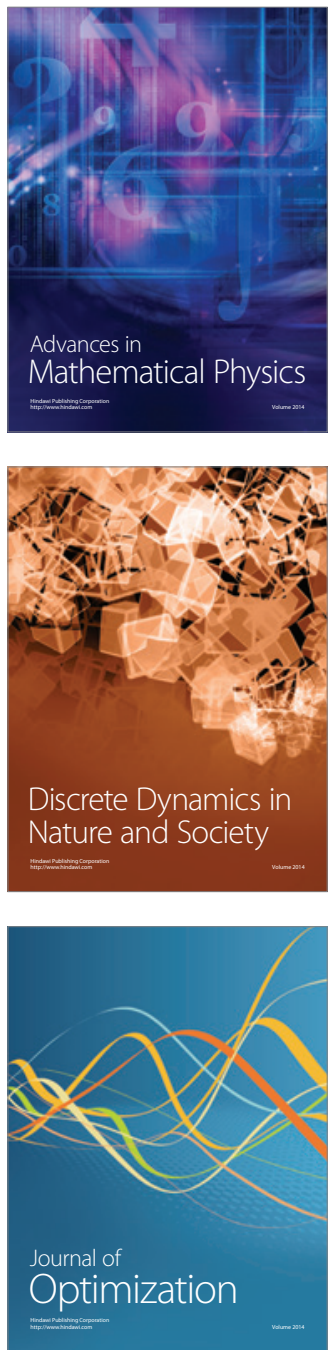